\title{
Avaliação do teor de antioxidantes, flavonoides e compostos fenólicos em preparações condimentares
}

MENDES, G.M.'; RODRIGUES-DAS-DORES, R.G.'; CAMPIDELI, L.C. ${ }^{1}$

1Universidade Federal de Ouro Preto, Ouro Preto, MG, CEP 35400-000, gabimm@msn.com, rosanagrd@ hotmail.com

RESUMO: Plantas condimentares são amplamente utilizadas na culinária como alimento funcional. Pacientes hipertensos, que retiram o sal de cozinha $(\mathrm{NaCl})$ de sua alimentação, relatam que os alimentos "perdem" o sabor. Visando alternativas para substituir o sal e melhorar a palatabilidade das preparações alimentares de pacientes hipertensos, o objetivo deste estudo foi desenvolver temperos à base de plantas medicinais e analisar os teores de compostos fenólicos, flavonoides e atividade antioxidante destes. Plantas (coentro, salsa, orégano, alecrim, manjericão, alho, cebola) cultivadas organicamente em Viçosa (MG) foram processadas e os temperos foram desenvolvidos a partir de combinação destas espécies, de acordo com teste aceitabilidade sensorial afetivo por meio de escala hedônica. Foram definidos três temperos, dos quais foram feitos extratos metanólicos por remaceração até esgotamento. Nas dosagens utilizou-se DPPH na avaliação da atividade antioxidante; reagente de FolinCiocalteu para compostos fenólicos e vanilina clorídrica para flavonoides. As análises foram feitas com quatro repetições e os resultados submetidos à análise de variância e teste de média a $5 \%$ de significância. Na aceitação subjetiva dos temperos a nota média foi $7,07 \pm 1,09$ e 8,0 $\pm 0,93$ na ação afetiva como alimento. $O$ teor mais elevado de compostos fenólicos foi no tempero 2 (alho, salsa e manjericão) $(55,04 \pm 4,307 \mathrm{mg} / \mathrm{mL})$, a maior ação antioxidante foi no tempero 1 (alho, cebola, coentro e orégano) nos tempos $0(48,72 \%)$ e 30 minutos $(78,37 \%)$. Os teores de flavonoides foram semelhantes nos 3 temperos. Todos os temperos tiveram boa aceitação por pacientes hipertensos e mostraram-se promissores na substituição dos condimentos convencionais.

Palavras-chave: plantas medicinais, temperos, hipertensos, análise sensorial, alimento funcional.

ABSTRACT: Evaluation of the content of antioxidants, flavonoids and phenolic compounds in culinary preparations. Plant herbs are widely used in cooking as functional food. Hypertensive patients, who remove salt $(\mathrm{NaCl})$ from their diets, report that the food "loses" flavor. Seeking alternatives to replace salt and improve the palatability of food preparations of hypertensive patients the aim of this study was to develop spices based on medicinal plants and analyze their contents of phenolic compounds, flavonoids and antioxidant activity. Plants (cilantro, parsley, oregano, rosemary, basil, garlic, onion) grown organically in the city of Viçosa (state of Mato Grosso, Brazil) were processed and the seasonings were developed from the combination of these species, according to the affective sensory acceptability test using the hedonic scale. Three spices were defined, and methanol extracts were made from them by maceration until exhaustion. In the dosages, we used DPPH to evaluate the antioxidant activity, Folin-Ciocalteu reagent for phenolic compounds and hydrochloric vanillin for flavonoids. The analyses were performed with four replicates and the results were subjected to analysis of variance and to a $5 \%$ significance test. In the subjective acceptance of the spices, the average score was $7.07 \pm 1.09$ and $8.0 \pm 0.93$ in the affective action as food. The highest content of phenolic compounds was for treatment 2 (garlic, parsley and basil) $(55.04 \pm 4.307 \mathrm{mg} / \mathrm{mL}$ ) and the highest antioxidant activity was measured in treatment 1 (garlic, onion, cilantro and oregano) at $0(48.72 \%)$ and 30 minutes $(78.37 \%)$. The contents of flavonoids were similar in the three treatments. All spices were well accepted by the hypertensive patients and have shown to be a promising replacement of conventional spices.

Key words: medicinal plants, spices, hypertension, sensory analysis, functional food. 


\section{INTRODUÇÃO}

A elevação da pressão arterial representa fator de risco para doença cardiovascular (DCV) (Nowson et al., 2003; SBC, 2010). Atualmente, aceita-se como alvo terapêutico a pressão arterial menor que 140/90 mmHg no consultório e menor que $135 / 85 \mathrm{mmHg}$ fora do consultório (Barochiner et al., 2012).

Vários são os fatores que podem estar associados à elevação da pressão arterial como sedentarismo, estresse, tabagismo, envelhecimento, história familiar, raça, gênero, peso e práticas alimentares inadequadas (Molina et al., 2003; SBC, 2010).

Dentre os fatores nutricionais que se associam à alta prevalência de hipertensão arterial está o elevado consumo de sódio (Rique et al., 2002; Molina et al., 2003; Nowson et al., 2003), prática habitual dos pacientes hipertensos que relatam que na retirada do sal $(\mathrm{NaCl})$ dos alimentos estes "perdem" o sabor. Desde 1985, a Organização Mundial de Saúde recomenda que a ingestão média diária de sal deva ser reduzida para $5 \mathrm{~g} / \mathrm{dia}$ ou menos, considerando o sódio oculto nos alimentos.

Inserido no contexto do tratamento não farmacológico dos portadores de hipertensão arterial, é preconizada a utilização das plantas em forma de chás, infusos e macerações, tanto na prevenção da elevação da pressão arterial, quanto na tentativa de redução dos valores pressóricos que já se encontram elevados (Oliveira \& Araújo, 2007). Alguns autores (Maia et al., 2011; Garcia Goméz e Sanchez-Muniz, 2000; Martín et al., 2004; Noldin et al., 2003; Ara tachjian et al., 2010; Cunha et al. 2003; Costa et al., 2006) relatam o uso de várias espécies tais como Rosmarinus officinalis L., Plectranthus barbatus And., Allium sativum L., Cynara scolymus L.; Leonurus sibiricus L. como hipotensoras discutindo os mecanismos de ação farmacológica e possíveis interações medicamentosas.

Desta forma, buscando melhorar a palatabilidade de pacientes hipertensos, novas opções de condimentos devem ser introduzidas nas preparações diárias para realçar o sabor e estimular hábitos alimentares saudáveis. Outro aspecto relevante na redução do consumo de sal por pacientes hipertensos é a inserção de temperos a base de plantas condimentares que possuem compostos farmacologicamente ativos, tais como terpenos ou óleos essenciais, compostos fenólicos e flavonoides caracterizando-as como alimentos funcionais.

O resgate das espécies aromáticas na culinária tradicional é de fácil aceitação pelos pacientes hipertensos. Os condimentos são mundialmente utilizados conferindo palatabilidade ou realçando características sensoriais nos alimentos (flavor), e, secundariamente, com finalidade de conservação, devido às propriedades antimicrobianas e antioxidantes.

Espécies consideradas especiarias, como orégano (Origanum vulgaris L.), alecrim (Rosmarinus officinalis L.) e manjericão (Ocimum basilicum L.) (Lamiaceae) destacam-se principalmente pela sua capacidade antioxidante (Kosar et al., 2005), no entanto, apenas o alecrim é amplamente utilizado em produtos alimentícios para conferir palatabilitade (De Morais, 2009).

$\mathrm{Na}$ família Apiaceae destacam-se o coentro (Coriandrum sativum L.) e a salsa (Petroselinum crispum (Mill.) Nym). Segundo estudos de Wong et al. (2006) os compostos fenólicos extraídos de ambos são responsáveis, em parte, por atividades antioxidantes. O extrato de salsa se mostrou responsável pelo aumentou significativo do volume urinário, confirmando o efeito diurético da planta (Kreydiyyeh et al., 2002). O extrato aquoso de $C$. sativum em cobaias induziu significativo aumento dose-dependente da diurese, excreção de sódio, cloreto e potássio, confirmando o uso tradicional de C. sativum como diurético (Aissaoui et al., 2007).

Nas Aliaceae, alho (Allium sativum L.) e cebola (Allium cepa L.) demonstram efeitos benéficos em doenças cardiovasculares por terem capacidade hipocolesterolêmica, hipolipidêmica, antihipertensiva, antidiabética, pró-ciculatória e antitrombótica (Craig, 1999; Mansoor, 2001; Amagase, 2006; Corzo-Martínez et al., 2007). A dose de alho sugerida para adultos é de $4000 \mathrm{mg}$ diários (Staba et al., 2001).

Este trabalho teve como objetivo desenvolver preparações (temperos) com plantas condimentares (coentro, salsa, orégano, alecrim, manjericão, alho, cebola) com ação hipotensora comprovada, analisar os teores de compostos fenólicos, flavonoides, atividade antioxidante e aceitação desta por pacientes hipertensos.

\section{MATERIAL E MÉTODO ou tratamento \\ Preparo e acondicionamento da amostra $40 \mathrm{~g}$ das espécies Allium cepa L. (cebola),} Allium sativum L. (alho), Coriandrum sativum L. (coentro), Ocimum basilicum L. (manjericão), Origanum vulgaris L. (orégano), Petroselinum crispum (Mill.) Nym. (salsa), Rosmarinus officinalis L. (alecrim), cultivadas organicamente e secas em estufa de ventilação forçada $\left(40^{\circ} \mathrm{C}\right)$, foram e moídas em moinho de facas, trituradas e rasuradas em gral de porcelana a fim de serem reduzidas a pó. Os pós das drogas vegetais foram acondicionados em embalagens aluminizadas e individuais, tipo sachês 
e selados termicamente (Figura 1).

As espécies foram testadas quanto sua palatabilidade, em combinações de ervas, buscando ter-se 3 temperos, que constituíram os tratamentos 1 (T1 - alho, cebola, coentro e orégano); tratamento 2 (T2 - manjericão, salsa e alho) e tratamento 3 (T3 - alho, cebola, coentro e alecrim), aos quais foram acrescentados de $1 \mathrm{~g}$ de sal com reduzido teor de sódio (hipossódico, 66\% menos sódio) (Figura 1).

A avaliação sensorial dos tratamentos ( $\mathrm{T} 1$, T2 e T3) foram feitas por voluntários normotensos e hipertensos, escolhidos aleatoriamente, que utilizaram os tratamentos em alimentos comuns do cotidiano sem nenhuma restrição ou prédeterminação. Cada avaliador recebeu 7 embalagens de cada um dos tratamentos, identificados por letras pré-codificadas por participante neutro, contendo 2 gramas de tempero, isto é 21 embalagens, para serem usados por 7 dias em uma das refeições diárias. O ensaio foi em duplo-cego e a avaliação foi por meio de método subjetivo com utilização de escala hedônica e escala de ação (IAL, 2008), onde foram atribuídas notas de 1 a 9 , de acordo com identificação codificada e a quantidade de tempero utilizada, aparência, aroma, sabor e a viabilidade de utilização (Figuras 2 e 3). Os dados foram submetidos à análise estatística descritiva quantitativa (ADQ), calculando-se dos respectivos desvios padrões e coeficientes de variação para validação dos tratamentos.

O projeto foi previamente aprovado em comitê de ética em pesquisa (CAAE 0023.0.238.00008) e juntamente com os temperos foi inserido a carta de apresentação, termos de esclarecimento (TE), termo de consentimento livre e esclarecido (TCLE).

\section{Preparo dos extratos}

$2 \mathrm{~g}$ de tratamentos T1, T2 e T3 foram extraídos com metanol PA (CROMOLINE) por extrações sucessivas (remaceração). Após 48 horas o extrato foi filtrado, reextraído sucessivamente até exaustão e seco em evaporador rotatório a pressão reduzida, pesado e o rendimento calculado. Todos os tratamentos foram feitos com quatro repetições.

Teste da capacidade antioxidante.

O ensaio da atividade antioxidante foi realizado utilizando o método de captura do radical 2,2-difenil-1-picrilhidrazil (DPPH) EF, EFL e ER e diluições a 400ppm (T1, T2 e T3), em quatro repetições, de acordo com ensaio realizado por Oliveira et al. (2011). Alíquotas de $0,1 \mathrm{~mL}$ dos extratos T1, T2 e T3 foram adicionados a 1,0mL de solução metanólica de DPPH (SIGMA) $(0,1 \mathrm{mM})$ realizando a leitura espectrofotométrica a $517 \mathrm{~nm}$ utilizando álcool metílico PA como branco (temperatura ambiente de $21^{\circ} \mathrm{C}$ ). No controle positivo utilizou-se 100ppm de $\mathrm{BHA}$ (hidroxianisol-butilado, SIGMA) (Jayaprakasha et al., 2002). O do consumo do radical DPPH foi calculado pela seguinte equação:

$\%$ AAT $=[($ AbsCONTROLE - AbsAMOSTRA $) /$ AbsCONTROLE] x 100

Onde: AbsAMOSTRA = Absorvância da solução de DPPH contendo extratos T1, T2 e T3; AbsCONTROLE = Absorvância da solução de $\mathrm{DPPH}$.

\section{Fenólicos}

Determinação do Teor de Compostos

O método utilizado foi adaptado de Singh et al. (2002), onde 0,2mg de extrato dos tratamentos T1, T2 e T3 foram adicionados a 2,5mL de reagente de Folin-Ciocalteu diluído (1:10) e 1,6mL de solução de carbonato de sódio a $7,5 \%$. As soluções são homogeneizadas e incubadas a $30^{\circ} \mathrm{C}$ durante 30 minutos, a temperatura de $24^{\circ} \mathrm{C}$. A absorbância foi medida a 765nm em espectrofotômetro (FEMTO). Curva padrão foi construída utilizando como padrão de referência, ácido tânico. Os resultados foram expressos em $\mathrm{mg} / \mathrm{mL}$ de ácido tânico.

\section{Determinação do teor de Flavonoides}

A $0,020 \mathrm{~g}$ do extrato de T1, T2 e T3 adicionou-se $2,5 \mathrm{~mL}$ de vanilina metanólica $(0,5 \%)$ e $2,5 \mathrm{ml}$ de solução dei com $\mathrm{HCl} 4 \%$. As soluções
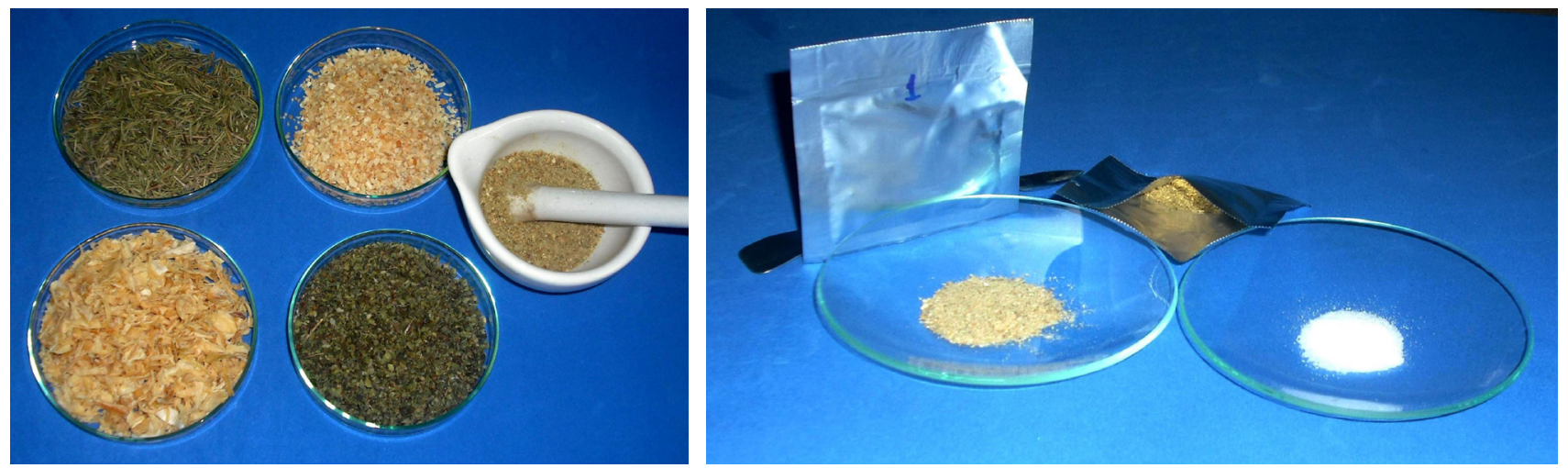

FIGURA 1. Preparo dos temperos. Arquivo pessoal. 2013. 
FIGURA 2. Ficha utilizada na avaliação da aceitação sensorial (escala hedônica) dos temperos. Ouro Preto, 2013.

Dados do Avaliador

\begin{tabular}{|c|c|c|c|}
\hline \multicolumn{2}{|l|}{ Nome } & \multirow{2}{*}{\multicolumn{2}{|c|}{\begin{tabular}{|l} 
Endereço \\
Outras doenças
\end{tabular}}} \\
\hline Idade & $\begin{array}{l}\text { Hipertenso } \\
\begin{array}{llll}\text { ( ) SIM } & \text { ( ) NÃO }\end{array}\end{array}$ & & \\
\hline \multicolumn{4}{|c|}{$\begin{array}{l}\text { Instruções } \\
\text { Você irá recebeu três amostras de temperos que serão usadas individualmente, em uma refeição. } \\
\text { Prove cuidadosamente cada uma e avalie, antes que a próxima seja utilizada. Represente o quanto gostou ou desgostou } \\
\text { de cada amostra, de acordo com escala do quadro. }\end{array}$} \\
\hline \multicolumn{4}{|l|}{ TEMPEROS } \\
\hline $\begin{array}{l}\text { TESTE DE } \\
\text { ESCALA HEDÔNICA }\end{array}$ & $\begin{array}{l}\text { Código } \\
\text { A1m2L }\end{array}$ & $\begin{array}{l}\text { Código } \\
\text { S2g3L }\end{array}$ & \begin{tabular}{|l|} 
Código \\
D1h2L
\end{tabular} \\
\hline \multicolumn{4}{|l|}{ Desgostei muitíssimo. } \\
\hline \multicolumn{4}{|l|}{ Desgostei muito. } \\
\hline \multicolumn{4}{|l|}{ Desgostei regulamente. } \\
\hline \multicolumn{4}{|l|}{ Desgostei ligeiramente. } \\
\hline \multicolumn{4}{|l|}{ Indiferente } \\
\hline \multicolumn{4}{|l|}{ Gostei ligeiramente. } \\
\hline \multirow{2}{*}{\multicolumn{4}{|c|}{ Gostei regulamente. }} \\
\hline & & & \\
\hline \multicolumn{4}{|l|}{$\begin{array}{l}\text { Gostei muito. } \\
\text { Gostei muitíssimo. }\end{array}$} \\
\hline
\end{tabular}

FIGURA 3. Ficha utilizada na avaliação da aceitação sensorial (escala de ação) dos temperos. Ouro Preto, 2013.

Dados do Avaliador

Nome

Idade

Instruções

Endereço

Outras doenças

Você irá recebeu três amostras que serão usadas individualmente, em uma refeição.

Prove cuidadosamente cada uma e avalie, antes que a próxima seja utilizada. Represente o quanto gostou ou desgostou de cada amostra, de acordo com escala do quadro.

Teste de aceitação

\section{TESTE DA ESCALA DE AÇÃO}

9. Eu comeria isto, em cada oportunidade que tivesse.

8. Eu comeria isto, muito frequentemente.

7. Eu comeria isto, frequentemente.

6. Eu comeria isto, agora e depois.

5. Eu comeria isto, se possível, mas não sairia da minha rotina.

4. Eu não gosto, mas se fosse preciso, comeria.

3. Eu comeria isto, raramente.

2. Eu comeria isto, se não tivesse outra escolha.

1. Eu comeria isto se fosse forçado.

Teste de aceitação

Quais refeições você realiza em casa?

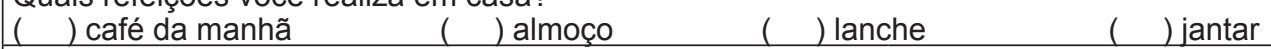

Quem é responsável pela preparação desses alimentos?

( ) eu mesma faço ( ) funcionário

() parentes ( ) outros ---

É viável a utilização do "tempero experimental" em algumas preparações do seu dia-a-dia?

( ) SIM ( ) NÃO

No seu dia-a-dia, em quais estes temperos poderiam ser usados?

Usualmente como costuma ser sua alimentação

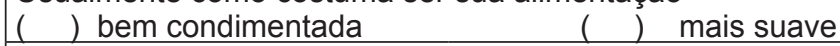

Acrescenta quais temperos nas refeições?

( ) SIM ( ) NÃO

Em que quantidade?

Como é a aceitação para tratamentos medicamentosos?

( ) Boa ( ) Ruim

( ) Tomo os medicamentos corretamente ( ) Não tomo os medicamentos

E modificações na dieta normal?

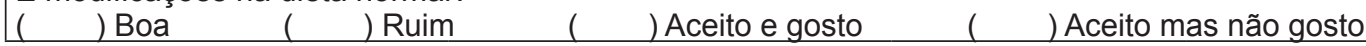

Rev. Bras. PI. Med., Campinas, v.17, n.2, p.297-304, 2015. 
foram homogeneizadas e protegidas da luz por 20 minutos, a temperatura ambiente $\left(24^{\circ} \mathrm{C}\right)$, procedeuse a leitura em espectofotômetro (FEMTO) a $500 \mathrm{~nm}$ (Jayaprakasha et al, 2002). A curva padrão foi construída utilizando rutina como substância de referência. Os resultados foram expressos em $\mu \mathrm{g} /$ $\mathrm{mL}$ de rutina.

\section{Análise estatística}

O delineamento experimental foi em blocos casualizados, todas as determinações foram realizadas em quatro repetições. $\mathrm{Na}$ análise sensorial dos tratamentos foi utilizado o Método Sensorial Descritivo por meio de Análise Descritiva Quantitativa (ADQ). Nas quantificações de compostos fenólicos, flavonoides, e atividade antioxidante, os dados foram submetidos à ANOVA e teste de média (Tukey) a $5 \%$ de significância utilizando o programa SAEG 9.1 for Windows (UFV, 2009).

\section{RESULTADO E DISCUSSÃO}

O controle de qualidade de preparações alimentares deve ser utilizado em cada etapa do processo de produção para garantir a qualidade e segurança alimentar, bem como para atender as expectativas e necessidades dos consumidores. O primeiro método para avaliar a qualidade dos produtos alimentares é a análise sensorial, que se baseia na utilização dos sentidos humanos, olfato, sabor e aparência destes.

Os resultados da análise sensorial dos tratamentos (T1, T2, e T3) avaliados por notas (escala de 1 a 9) expressando o gostar ou desgostar (escala hedônica) transformados em análise de frequência tiveram na aceitação subjetiva dos temperos a nota média de $7,7 \pm 1,09(85,55 \%)$ e $8,0 \pm 0,38(88,89 \%)$ na ação afetiva como alimento (Tabela 1).

$\mathrm{Na}$ escala de ação, $70 \%$ dos avaliadores atribuíram notas superiores a 6 ( $>6=$ eu comeria isto, agora e depois) para T1, T2 e T3; $5 \%$ dos avaliadores atribuíram nota igual a 5 (eu comeria isto, se possível, mas não sairia da minha rotina) para $\mathrm{T} 1$ e $10 \%$ atribuíram duas notas menores que 5 para T2 e T3.

A escala de aceitação subjetiva analisou apenas o quesito paladar agradável numa escala de gostei muitíssimo a desgostei muitíssimo, a variação obtida mostrou que a aceitação foi boa onde $85 \%, 80 \%$ e $75 \%$ dos avaliadores atribuíram notas maiores que 7 (gostei regularmente) para T1, T2 e T3 respectivamente.

$\mathrm{Na}$ escala de ação afetiva que investigou a utilização da preparação, em caso de alguma patologia, o resultado obtido foi bastante satisfatório, uma vez que a maioria dos avaliadores hipertensos aprovou os temperos e afirmaram que os usariam frequentemente nas preparações diárias.

As combinações dos temperos estão dispostas na tabela 1. Temperos com sabor mais familiar, como os que continham manjericão e alecrim são mais facilmente identificados e relacionados com certa preparação (ex: massas, carnes). Avaliadores masculinos preferiram às preparações com coentro e orégano. Quanto às sugestões de utilização no diaa-dia, os alimentos líquidos (sopas, caldos, feijão) foram os mais referenciados $(57 \%)$, seguidos de carnes $(19 \%)$, saladas $(14 \%)$ e outros $(10 \%)$. Houve grande aceitação para uso em lanches ou refeições ligeiras (pipoca, mandioca cozida).

Os rendimentos dos extratos secos foram de $25,33 \pm 0,07 \%$ em T1, 25, $13 \pm 0,04 \%$ em T2 e $25,35 \pm 0,10 \%$ em T3. Os rendimentos médios foram de $25,27 \pm 0,12 \%$. Os tratamentos T1 e T2 tiveram rendimentos semelhantes, o que era esperado devido à proximidade das combinações de condimentos (alho, cebola e coentro) na preparação, diferindo de T2 (Tabela 2).

A equação de calibração de compostos fenólicos foi $\hat{y}=0,0077 x-0,0034\left(r_{2}=0,991\right)$. Os valores médios de compostos fenólicos foi de $51,23 \pm 4,64 \mathrm{mg} / \mathrm{mL}$. O teor mais elevado de compostos fenólicos foi em T2 $(55,04 \pm 4,307 \mathrm{mg} / \mathrm{mL})$ (Tabela 2), o que mostra maior eficácia no potencial redutor de diminuição da pressão.

A equação da reta de doseamento de flavonoides foi $\hat{y}=0,000005 x+0,0144,\left(r_{2}=0,9878\right)$. O teor médio de $604,22 \pm 25,80 \mu \mathrm{g} / \mathrm{mL}$. Os teores de flavonoides foram semelhantes nos 3 tratamentos (Tabela 2), o que nos permite inferir que não há diferença de eficácia no uso dos diversos

TABELA 1. Composição percentual dos tratamentos T1, T2 e T3 em função dos condimentos utilizados e escala de aceitação em notas (1 a 9). Ouro Preto, 2013.

\begin{tabular}{lccc}
\hline & \multicolumn{3}{c}{ TRATAMENTO } \\
CONDIMENTO & T1 & T2 & T3 \\
Alho & $30 \%$ & $40 \%$ & $40 \%$ \\
Cebola & $30 \%$ & - & $30 \%$ \\
Coentro & $10 \%$ & - & $10 \%$ \\
Salsa & - & $30 \%$ & - \\
Manjericão & - & $30 \%$ & - \\
Orégano & $30 \%$ & - & - \\
Alecrim & & - & $20 \%$ \\
Escala hedônica ns & 7,85 & 7,69 & 7,69 \\
CV (\%) = 14,15\% & & & \\
Escala afetivans & 7,95 & 7,65 & 8,41 \\
CV $(\%)=23,21 \%$ & & & \\
\hline
\end{tabular}

ns dados não diferiram significativamente a $1 \%$ de probabilidade pelo teste de Tukey. 
TABELA 2. Teores médios de rendimento dos extratos (REND) (\%), de compostos fenólicos (CF) em gramas por mililitros $(\mathrm{mg} / \mathrm{mL})$, de flavonoides em microgramas por mililitros $(\mu \mathrm{g} / \mathrm{mL})$, do percentual da atividade antioxidante (\% AAT), nos tempos zero (AT 0) e trinta minutos (AT 30) nos tratamentos T1 (Orégano, alho, cebola e coentro), T2 (Manjericão, salsa e alho) e T3 (Alecrim, algo, cebola e coentro). Ouro Preto, 2013.

\begin{tabular}{|c|c|c|c|c|c|}
\hline \multirow{3}{*}{ TRATAMENTOS } & \multicolumn{5}{|c|}{ MÉDIAS } \\
\hline & \multirow[b]{2}{*}{ REND* } & \multirow[b]{2}{*}{$\mathrm{CF}^{*}$} & \multirow[b]{2}{*}{ FLAV ns } & \multicolumn{2}{|c|}{ AAT* $^{*}$} \\
\hline & & & & AT 0 & AT 30 \\
\hline T1 & $25,32 \mathbf{A}$ & $50,79 \mathrm{AB}$ & 622,00 & $48,71 \mathrm{~A}$ & $78,37 \mathbf{A}$ \\
\hline T2 & $25,13 \mathrm{~B}$ & $55,03 \mathbf{A}$ & 592,00 & 9,67 B & $22,88 \mathrm{~B}$ \\
\hline T3 & $25,35 \mathrm{~A}$ & 47,86 В & 598,66 & $7,10 \mathrm{~B}$ & $12,91 \mathbf{B}$ \\
\hline CV (\%) & 0,30 & 7,31 & 3,90 & 21,99 & 33,25 \\
\hline
\end{tabular}

As médias seguidas de uma mesma letra não variaram estatisticamente pelo teste de Tukey a $5 \%$ de probabilidade.

ns. Não significativo ao nível de $5 \%$ de probabilidade.

* Significativo ao nível de $5 \%$ de probabilidade.

condimentos utilizados, no que tange ao teor médio de flavonoides. A importância de teores de flavonoides semelhantes nesta preparação está diretamente interligada na capacidade de prevenção de danos cardiovasculares, redução dos níveis glicêmicos e colesterolêmicos (Dornas et al, 2007). Evidências epidemiológicas crescentes sugerem que há correlação negativa entre consumo de alimentos ou bebidas ricas em polifenóis e a incidência de doenças cardiovasculares (Rein et al., 2000).

Os teores médios de antioxidantes nos temperos, no tempo zero (t0), foram de 21,83 \pm $20,10 \%$ e após 30 minutos (t30) de $38,06 \pm 31,92 \%$. $A$ atividade antioxidante mais elevada ocorreu em T1 (alho, cebola, coentro e orégano) $(48,72 \%)$ $(78,37 \%)$, nos tempos 0 e 30 minutos (Tabela 2). Resultados superiores a $60 \%$ demonstram excelente poder redutor e atuação no combate aos radicais livres que previne doenças ateroscleróticas (Miliauskas et al, 2004).

Alho foi utilizado em $30 \%$ em T1, $40 \%$ em T2 e 40\% em T3, o que auxiliou nos altos índices de compostos fenólicos em T2 e de antioxidantes em T1. As propriedades antioxidantes do alho podem ser atribuídas aos compostos fenólicos e aos compostos sulfurados. Destacam-se y-glutamil-Salil-L-cisteína e S-alil-L-cisteina sulfóxidos (alinina). Os sulfóxidos, exceto cicloalinina, são convertidos em tiossulfatos (como alicina) por reação enzimática quando o bulbo do alho é processado, cortado ou amassado (Amagase, 2006; Staba et al., 2001). A dose terapêutica de alho sugerida a adultos é de 4000 mg diários (Staba et al., 2001).

Segundo o estudo de Guerra et al. (2005), a ação antioxidante demonstrada pelo extrato etéreo do coentro, prevê possibilidade de ser fonte de substâncias bioativas. O extrato aquoso de coentro (Coriandrum sativum), com infusão intravenosa em ratos induziu significativo aumento dose-dependente da diurese, excreção de sódio, cloreto e potássio. Confirmando o uso tradicional de $C$. sativum como diurético (Aissaoui et al., 2007), corroborado pelo maior rendimento de T1 no qual há $10 \%$ de coentro.

Alho (Allium sativum) e a cebola (Allium cepa) demonstram efeitos benéficos sobre enfermidades cardíacas, através da redução dos níveis de colesterol total e LDL, aumento do colesterol HDL e diminuição da peroxidação lipídica (Almeida \& Suyenaga, 2009). Tanto o alho quanto a cebola estão presentes nas preparações T1 e o alho apenas presente na preparação $\mathrm{T} 2$, o que reforça os benefícios dessas preparações no combate às doenças cardiovasculares por serem constituídos por altas concentrações de antioxidantes e compostos fenólicos.

Coentro (Coriandrum sativum), presente em T1 e T3 e a Salsa (Petroselinum crispum) presente em T2 são responsáveis por atividades antioxidantes, segundo estudos de Wong et al. (2006). O mesmo estudo comprova que a suplementação de dietas com folhas frescas de salsa pode aumentar a capacidade antioxidante no plasma de ratos e levar a diminuição do estresse oxidativo em humanos (Wong et al., 2006).

Salsa (Petroselinum crispum) é amplamente utilizada na medicina popular brasileira como diurético (De Campos et al., 2009). O extrato de salsa também se mostrou responsável pelo aumento significativo do volume urinário, confirmando, assim, o efeito diurético da planta. Na determinação deste local de ação no organismo, a bomba $\mathrm{Na}^{+} / \mathrm{H}^{+} \mathrm{e} o$ canal simporte $\mathrm{NaKCl}_{2}$ foram inibidos. Apesar de tanto a salsa quanto o coentro possuírem atividade antioxidante, no presente estudo os resultados de coentro sobressaíram em relação à salsa uma vez que em T1 em que há coentro na preparação foi encontrado maior potencial antioxidante em comparação com T2 que contém salsa.

Manjericão (Ocimum $\mathrm{sp}$ ) possui elevado poder antioxidante (Pitaro et al., 2012), sendo muito utilizadas como condimento. Nesse estudo verificou-se que o manjericão, presente em T2, é rico em compostos fenólicos, principais compostos 
que possuem grupamentos $\mathrm{OH}$ - que funcionam como sequestradores de radicais agindo tanto na etapa de iniciação como na propagação do processo oxidativo (Soares, 2002).

A boa aceitação dos produtos motiva novos testes, comprova a facilidade de uso, e permite a incorporação dos temperos/ ervas medicinais nos hábitos alimentares dos hipertensos, facilitando a aceitação/ consumo e palatabilidade dos alimentos, conforme mostrado nos resultados da tabela 1. A partir dos resultados obtidos conclui-se que preparações com alto teor de compostos fenólicos e antioxidantes são provavelmente mais eficazes na atuação em distúrbios cardiovasculares e na prevenção do envelhecimento celular.

A preparação condimentar, constituída por alho, salsa e manjericão, teve maior teor de compostos fenólicos e a que contém alho, cebola, coentro e orégano possui maior atividade antioxidante, que confere ação especial no combate aos radicais livres. Todos os temperos foram bem aceitos por pacientes hipertensos. Em função dos resultados obtidos outros estudos podem ser feitos para avaliar a influência dos temperos nos preditores de obesidade, nos parâmetros sanguíneos e na hipertensão arterial de portadores de doenças cardiovasculares.

\section{REFERÊNCIAS}

AISSAOUI, A.; EL-HILALY, J.; ISRAILI, Z.H.; LYOUSSI, B. Acute diuretic effect of continuous intravenous infusion of an aqueous extract of Coriandrum sativum L. in anesthetized rats. Journal of Ethnopharmacology, v.115, n.1, p.89-95, 2007.

ALMEIDA, A.; SUYENAGA, E.S. Ação farmacológica do alho (Allium sativum L.) e da cebola (Allium cepa L.) sobre o sistema cardiovascular: revisão bibliográfica. Revista da Sociedade Brasileira de Alimentação e Nutrição. São Paulo, v.34, n.1, p.185-197, 2009.

AMAGASE, H. Clarifying the Real Bioactive Constituents of Garlic. Journal of Nutrition, v. 136, n.3, p.716-725, 2006. Disponível em: http://jn.nutrition.org. Acesso em: 23 maio 2013.

ARA TACHJIAN, M.D.; VIQAR MARIA, M.B.B.S.; ARSHAD JAHANGIR, M.D. Use of herbal products and potential interactions in patients with cardiovascular diseases. Journal of the American College of Cardiology, v.55, n.6, p.515-525, 2010.

BAROCHINER, J.; APARICIO, J.A.L.; MORALES, M.; CUFFARO, P.E.; RADA, M.; GALARZA, C.; INGARAMO, R.; RESK, J.; VILLAMIL, A.; BORREGO, C.; ZILBERMAN, J.; WAISMAN, G. Evaluación del tratamiento anti-hipertensivo a través del monitoreo domiciliario de la presión arterial (MDPA). Revista del Hospital Italiano de Buenos Aires, v.32, n.1, p.9-13. 2012.

CORZO-MARTÍNEZ, M.; CORZO, N.; VILLAMIEL, M. Biological properties of onions and garlic. Food Science
\& Technology, v.18, p.609-625, 2007.

COSTA, M.C.C.D. Uso popular e ações farmacológicas de Plectranthus barbatus Andr.(Lamiaceae): revisão dos trabalhos publicados de 1970 a 2003. Revista Brasileira de Plantas Medicinais, v.8, n.2, p.81-88, 2006.

CRAIG, W.J. Health-promoting properties of common herbs. The American Journal of Clinical Nutrition, n.70, p.491S-499S, 1999. Disponível em: www.ajcn. org. Acesso em: 02 out. 2012.

CUNHA, A.P.; SILVA, A.P.; ROQUE, O.R. Plantas e produtos vegetais em fitoterapia. Lisboa: Fundação Calouste Gulbenkian, 2003. 701p.

DE CAMPOS, K.E.; BALBI, A.P.C.; ALVES, M.J.Q.F. Diuretic and hipotensive activity of aqueous extract of parsley seeds (Petroselinum sativum Hoffm.) in rats. Revista Brasileira de Farmacognosia, v.19, n.1a, p.41-45, 2009.

DE MORAIS, S.M.; CAVALCANTI, E.S.B.; COSTA, S.M.O.; AGUIAR, L.A. Ação antioxidante de chás e condimentos de grande consumo no Brasil. Brazilian Journal of Pharmacognosy, v.19, n.1b, p.315-320, 2009.

DORNAS, W.C.; OLIVEIRA, T.T.; RODRIGUES-DASDORES, R.G.; SANTOS, A.F.; NAGEM, T.J. Flavonoides: potencial terapêutico no estresse oxidativo. Revista de Ciências Farmacêuticas Básica e Aplicada, v.28, n.3, p.241-249, 2007.

GARCIA GOMEZ, L.J; SANCHEZ-MUNIZ, F.J. Revisión: Efectos cardiovasculares del ajo (Allium sativum). ALAN, v.50, n.3, p.219-229, 2000.

GUERRA, N.B.; MELO, E.A.; FILHO, J.M. Antioxidant compounds from coriander (Coriandrum sativum L.) etheric extract. Journal of Food Composition and Analysis, v.18, p.193-199, 2005. Disponível em: www. elsevier.com/locate/jfca. Acesso em: 20 out. 2012.

JAYAPRAKASHA, G.K.; CHIDAMBARA MURTHY, K.N.; SINGH, R.P. Antioxidant activities of grape (Vitis vinifera) pomace extracts. Journal of Agricultural and Food Chemistry, v.50, n.21, p.5909-5914, 2002.

KOSAR, M.; DORMAN, H.J.D.; HILTUNEN, R. Effect of an acid treatment on the phytochemical and antioxidant characteristics of extracts from selected Lamiaceae species. Food Chemistry, v.91, p.525-533, 2005. Disponível em: www.elsevier.com/locate/foodchem. Acesso em: 10 maio 2013.

KREYDIYYEH, S.I., USTA, J. Diuretic effect and mechanism of action of parsley. Journal of Ethnopharmacology, v.79, p.353-357, 2002. Disponível em: www.elsevier. com/locate/jethpharm. Acesso em: 15 junho 2012.

MAIA, L.F.; CASTRO, Q.J.T; RESENDE, F.M.F.; RODRIGUES-DAS-DORES, R.G. Plantas medicinais e hipertensão. Farmácia Revista, v.1, n.24, p.24-25, 2011. Disponível em http://www.crfmg.org.br/ novosite/ index.php/farmacia-revista. Acesso em: 20 jan. 2014.

MANSOOR, G.A. Herbs and alternative therapies in the hypertension clinic. American Journal of Hypertension, v.14, p.971-975, 2001. Disponível em: http://www.sciencdirect.com. Acesso em: 03 nov. 2013.

MARTÍN, L.S.M.M. et al. Actividad diurética y antipirética de un extracto fluido de Rosmarinus officinalis L. em ratas. Rev. Cubana Plant. Med., v.9, n.1, p.0-0. 2004. Disponível em: http://scielo.sld.cu/scielo.phpscript=sci_ 
arttext\&pid=S1 028-47962004000100007\&lng=es\&nrm =iso. Acesso em: 17 março 2014.

MOLINA, M.C.B.; CUNHA, R.S.; HERKENHOFF, L.F.; MILL, J.G. Hipertensão arterial e consumo de sal em população urbana. Revista de Saúde Pública, v.37, n.6, p.743-750, 2003.

NOLDIN, V.F., MONACHE, F.D., YUNES, R.A. Composição química e atividade biológica de Cynara scolymus $\mathrm{L}$. cultivada no Brasil. Química Nova, v.26, n.3, p.331334, 2003.

IAL. INSTITUTO ADOLFO LUTZ. Métodos Físicoquímicos para análise de alimentos. São Paulo: Instituto Adolfo Lutz, p. 1020, 2008.

MILIAUSKAS, G.; VENSKUTONIS, P.R.; VAN BEEK, T.A. Screening of radical scavenging activity of some medicinal and aromatic plant extracts. Food Chem., v. 85, n.2, p. 231-237, 2004.

NOWSON, C.A.; MORGAN, T.O.; GIBBONS, C. Decreasing dietary sodium while following a selfselected potassium-rich diet reduces blood pressure. Jornal de Nutrição, v.133, p.4118-4123, 2003. Disponível em: http://jn.nutrition.org. Acesso em: 22 mai. 2013.

OLIVEIRA, C.J.; ARAÚJO, T.L. Plantas medicinais: usos e crenças de idosos portadores de hipertensão arterial. Revista Eletrônica de Enfermagem, v.9, n.1, p.93105, 2007. Disponível em: www.fen.ufg.br/revista/v9/ n1/v9n1a07.htm. Acesso em: 10 dez. 2011.

PITARO, S.P.; FIORANI, L.V.; JORGE, N. Potencial antioxidante dos extratos de manjericão (Ocimum basilicum Lamiaceae) e orégano (Origanum vulgare Lamiaceae) em óleo de soja. Revista Brasileira de Plantas Medicinais, v.14, n.4, p.686-691, 2012.

REIN, D.; PAGLIERONI, T.G.; PEARSON, D.A.; WUN,
T.; SCHMITZ, H.H.; GOSSELIN, R.; KEEN, C.L. Cocoa and wine polyphenols modulate platelet activation and function. Jornal de Nutrição, v.130, n.8, p.2120S2126S, 2000.

RIQUE, B.R.; SOARES, E.A.; MEIRELLES, C.M. Nutrição e exercício na prevenção e controle das doenças cardiovasculares. Rev. Bras. Med. Esporte, v.8, p. 244254, 2002. Disponível em: www.scielo.com.br. Acesso em: 12 ago. 2011.

UFV. SAEG 9.1 for Windows: Sistema de análises estatísticas e genéticas. Programa de computador. Viçosa: UFV. 2009.

SINGH, R.P.; MURTHY, C.; JAYAPRAKASHA, G.K. Studies on the antioxidant activity of pomegranate (Punica granatum) peel and seed extracts using in vitro models. Journal of Agricultural and Food Chemistry, v.50, p.81-86, 2002.

SOARES, S. E. Ácidos fenólicos como antioxidantes. Revista de Nutrição, v.15, n.1 p.71-81, 2002

STABA, E.J.; LASH, L.; STABA, J.E.A. Commentary on the effects of garlic extraction and formulation on product composition. Jornal de Nutrição, v.131, n.3, p.1118S-1119S, 2001. Disponível em: http://jn.nutrition. org. Acesso em: 21 maio 2013.

SBC. Sociedade Brasileira de Cardiologia/ Sociedade Brasileira de Hipertensão/ Sociedade Brasileira de Nefrologia. VI Diretrizes Brasileiras de Hipertensão. Arq Bras Cardiol; v.95,n.1, p.1-51, 2010.

WONG, P.Y.Y.; KITTS, D.D. Studies on the dual antioxidant and antibacterial properties of parsley (Petroselinum crispum) and cilantro (Coriandrum sativum) extracts. Food Chemistry, v.97, p.505-515, 2006. Disponível em: www.elsevier.com/locate/foodchem/. Acesso em: 10 nov. 2013. 